\title{
Left ventricular flow patterns in healthy subjects and patients with prosthetic mitral valves: An in vivo study using echocardiographic particle image velocimetry
}

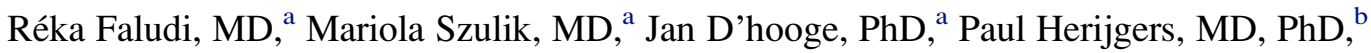 \\ Frank Rademakers, $\mathrm{MD}, \mathrm{PhD},{ }^{\mathrm{a}}$ Gianni Pedrizzetti, $\mathrm{PhD},{ }^{\mathrm{c}}$ and Jens-Uwe Voigt, $\mathrm{MD}, \mathrm{PhD}^{\mathrm{a}}$
}

\begin{abstract}
Objective: Echocardiographic particle image velocimetry is a new feature tracking-based approach to visualize and quantify left ventricular flow patterns in vivo. We investigated the potential role of this new technique by assessing vortex formations in healthy left ventricles and the effect of different types of prosthetic valves on intraventricular flow patterns and flow-mediated energy dissipation.
\end{abstract}

\begin{abstract}
Methods: We examined 19 patients (mean age, $57 \pm 19$ years; 10 women). Nine were healthy, and 10 had prosthetic mitral valves ( 5 had bileaflet valves, 4 had bioprostheses, and 1 had a tilting-disc valve). Boluses of left heart contrast were administered intravenously. Echocardiographic apical views were analyzed offline by using prototype software that allowed intracavitary flow to be explored and enabled calculations of energy dissipation (relative pulsatile vorticity strength and vortex pulsation correlation) by means of particle image velocimetry.
\end{abstract}

Results: In healthy hearts a vortex filling the entire ventricle stores the kinetic energy of the blood and smoothly redirects the blood to the outflow tract. In patients with prosthetic valves, completely different flow patterns were identified depending on the type, orientation, and position of the valves, as well as left ventricular geometry. Patients with prosthetic valves showed significantly higher left ventricular energy dissipation than healthy subjects (relative pulsatile vorticity strength, $2.4 \pm 0.7$ vs $1.6 \pm 0.4[P<.001]$; vortex pulsation correlation, $1.2 \pm 0.5$ vs $0.7 \pm 0.2[P<.001])$.

Conclusions: Echocardiographic particle image velocimetry is feasible. It clearly distinguishes flow patterns in healthy hearts from those in hearts with different types of prosthetic valves. Echocardiographic particle image velocimetry offers new insights into cardiac function and might be of importance to optimize valve replacement therapy. (J Thorac Cardiovasc Surg 2010;139:1501-10)

Flow can be described as laminar, vortical, or turbulent. Although laminar flow, which can occur in straight blood vessels, is characterized by parallel stream lines, vortical and turbulent flows are dominated by swirling motion of the fluid at different scales. In the 15th century, Leonardo da Vinci pictured vortices in the sinuses above the aortic valve. ${ }^{1}$ Until today, vortices in the human circulation remain a challenging aspect of fluid dynamics. Modern phase-contrast magnetic resonance imaging (MRI) has shown that vortical flow is a common finding in the human heart, ${ }^{2-6}$ as well as in the great arteries. $^{7,8}$

Vortices are created when the boundary layer of a fluid detaches from a sharp edge. ${ }^{9}$ In the left ventricle laminar mitral

\footnotetext{
From the Departments of Cardiology and Cardiac Surgery, ${ }^{\mathrm{b}}$ University Hospital Gasthuisberg, Catholic University Leuven, Leuven, Belgium, and the Department of Civil Engineering, ${ }^{\mathrm{c}}$ University of Trieste, Trieste, Italy.

Disclosures: None.

Supported in part by the Flemish government (Fonds Wetenschappelijk Onderzoek No.G068508 N).

Received for publication Dec 5, 2008; revisions received June 10, 2009; accepted for publication July 6, 2009; available ahead of print April 5, 2010.

Address for reprints: Jens-Uwe Voigt, MD, PhD, Department of Cardiology, University Hospital Gasthuisberg Cath. University Leuven, Herestraat 49, 3000 Leuven, Belgium (E-mail: jens.uwe.voigt@gmx.net).

$0022-5223 / \$ 36.00$

Copyright (c) 2010 by The American Association for Thoracic Surgery

doi:10.1016/j.jtcvs.2009.07.060
}

inflow is converted into a vortex formation at the tips of the mitral valve leaflets. This vortex formation maintains the momentum of the blood and allows smooth redirection of blood flow toward the outflow tract during systole with minimal generation of turbulence, thus avoiding large losses of kinetic energy. ${ }^{5,10,11}$

Vortical flow can be characterized by the quantity vorticity $(\omega)$ as follows:

$$
\omega=\frac{\Delta v_{y}}{\Delta v_{x}}-\frac{\Delta v_{x}}{\Delta v_{y}} .
$$

In a 2-dimensional flow field vorticity can be calculated as the difference of the gradient of the y-component of the flow velocity in $\mathrm{x}$-direction $\left(\frac{\Delta v_{y}}{\Delta v_{x}}\right)$ and the gradient of the $\mathrm{X}$ component of the flow velocity in the y-direction $\left(\frac{\Delta v_{y}}{\Delta v_{x}}\right)$ Therefore vorticity is positive with counterclockwise vortex rotation and negative with clockwise rotation. Laminar flow has a zero vorticity (Figure 1). ${ }^{9}$

Because of fluid viscosity, a vortex loses energy. This energy loss is particularly high if there are rapid changes in vorticity (high pulsatility) or if many small vortices interact (turbulence). In other words, a single, large, and steady vortex is an energetically favorable condition, whereas splitting the bloodstream into smaller, rapidly changing vortices is 


\section{Abbreviations and Acronyms \\ MRI = magnetic resonance imaging \\ PIV $=$ particle image velocimetry \\ RS = relative pulsatile vorticity strength \\ $\mathrm{VPC}=$ vortex pulsation correlation \\ $\mathrm{VRS}=$ vortex relative pulsatile vorticity strength}

unfavourable ${ }^{9}$ : kinetic energy gets lost, which needs to be provided again by the ventricular musculature.

Changes in regional vorticity can be used to estimate energy dissipation in the fluid. They can be expressed relative to the steady vorticity component to obtain a dimensionless parameter. This relative strength of the pulsatile component of vorticity can be calculated for the entire ventricle (relative pulsatile vorticity strength $[R S]$ ) or for the vortex region alone (vortex relative pulsatile vorticity strength [VRS]). RS and VRS are defined as follows:

$$
\begin{gathered}
R S=\frac{\int_{L V} \omega_{1}(x, y) d x d y}{\int_{\text {vortex }} \omega_{0}(x, y) d x d y} \\
V R S=\frac{\int_{\text {vortex }} \omega_{1}(x, y) d x d y}{\int_{\text {vortex }} \omega_{0}(x, y) d x d y},
\end{gathered}
$$

where $\omega_{0}$ represents the steady and $\omega_{1}$ represents the pulsatile component (first harmonic) of regional vorticity. ${ }^{9}$

Conceptually similar but with a different mathematic definition, the vortex pulsation correlation (VPC) can be calculated as follows:

$$
V P C=\frac{A_{\text {vortex }} \int_{\text {vortex }} \omega_{0}(x, y) \omega_{1}(x, y) d x d y}{\left(\int_{\text {vortex }} \omega_{0}(x, y) d x d y\right)^{2}},
$$

where $\omega_{0}$ represents again the steady and $\omega_{1}$ represents the pulsatile component (first harmonic) of regional vorticity and $A_{\text {vortex }}$ represents the vortex area. ${ }^{9}$

The transvalvular flow characteristics of commonly used prosthetic mitral valves are well known. ${ }^{12}$ However, the subsequent changes of intraventricular flow patterns, particularly vortex formation, in relation to the type and orientation of the mitral valve prosthesis are difficult to assess in vivo and have not been characterized. Several factors influencing outcome in mitral valve replacement have been reported. ${ }^{13,14}$ The extent to which intracavitary blood flow patterns contribute has not been able to be measured and is thus yet to be determined.
Traditional spectral or color Doppler echocardiography measures velocities relative to the transducer. Doppler data therefore contain only 1-dimensional information on the blood velocity component toward or away from the transducer and do not allow assessment of the direction of blood flow.

Particle image velocimetry (PIV) is a well-known principle of determining the velocity and direction of fluid streams by analyzing the change in position of small particles that drift with the fluid to be investigated. ${ }^{15}$ With the recent development of echocardiographic speckle-tracking technology, it is now possible to apply this approach to contrast-enhanced echocardiographic imaging. ${ }^{16-18}$

In this study we use the new echocardiographic PIV method to investigate the influence of mitral valve replacement therapy on blood flow patterns in the left ventricular cavity.

\section{MATERIALS AND METHODS \\ Study Population}

We examined 19 patients (mean age, $57 \pm 19$ years; 10 women). Nine were healthy volunteers without signs or symptoms of heart disease. Ten patients had prosthetic mitral valves ( 5 bileaflet valves [29-33 mm, St Jude; St Jude Medical, Inc, St Paul, Minn] in anatomic orientation, 4 bioprostheses [29-33 mm, Carpentier-Edwards; Edwards Lifesciences, Irvine, Calif], and 1 tilting-disc valve [Medtronic Hall, $29 \mathrm{~mm}$; Medtronic, Minneapolis, Minn] with anterior orientation of the greater orifice). Patients with atrial fibrillation, segmental wall motion abnormalities, or impaired left ventricular systolic function (regional wall motion abnormalities or ejection fraction $<50 \%$ ) were not included. Six patients had medically treated arterial hypertension. The local ethics committee approved the study. All subjects provided written informed consent before inclusion.

\section{Echocardiographic Image Acquisition}

Subjects were imaged in a supine position by using an Acuson Sequoia C512 ultrasound system (Siemens Medical Solutions, Mountain View, Calif). In addition to a standard echocardiogram, 2-dimensional grayscale images were acquired with high frame rate (80-100/s) in all 3 standard apical views and repeated intravenous administration of a low dose of left heart contrast $(0.1-0.2 \mathrm{~mL}$ of SonoVue). The mechanical index was set to 0.5 to 0.8 to avoid apical contrast bubble destruction. Images were acquired when cavity contrast distribution was homogeneous and single contrast bubbles could be distinguished. Image loops of 3 cardiac cycles were digitally stored for subsequent offline analysis.

\section{Data Analysis}

Conventional 2-dimensional and Doppler recordings were analyzed during the examination or with a dedicated workstation (Syngo, Siemens Medical Solutions). We measured left ventricular volumes and ejection fractions using a biplane Simpson's approach, as well as left ventricular and left atrial dimensions and the prosthetic mitral valve gradient.

\section{Particle Image Velocimetry}

Echocardiographic loops were processed offline by using a dedicated prototype software (Omega Flow Version 2.3.1., Siemens). At first, the endocardial border was manually traced in one still frame and then automatically tracked by the software during the cardiac cycle. In a second step the so-defined cavity area was analyzed with a feature tracking algorithm. Contrast bubbles were assumed to move with the blood flow, and thus tracking 


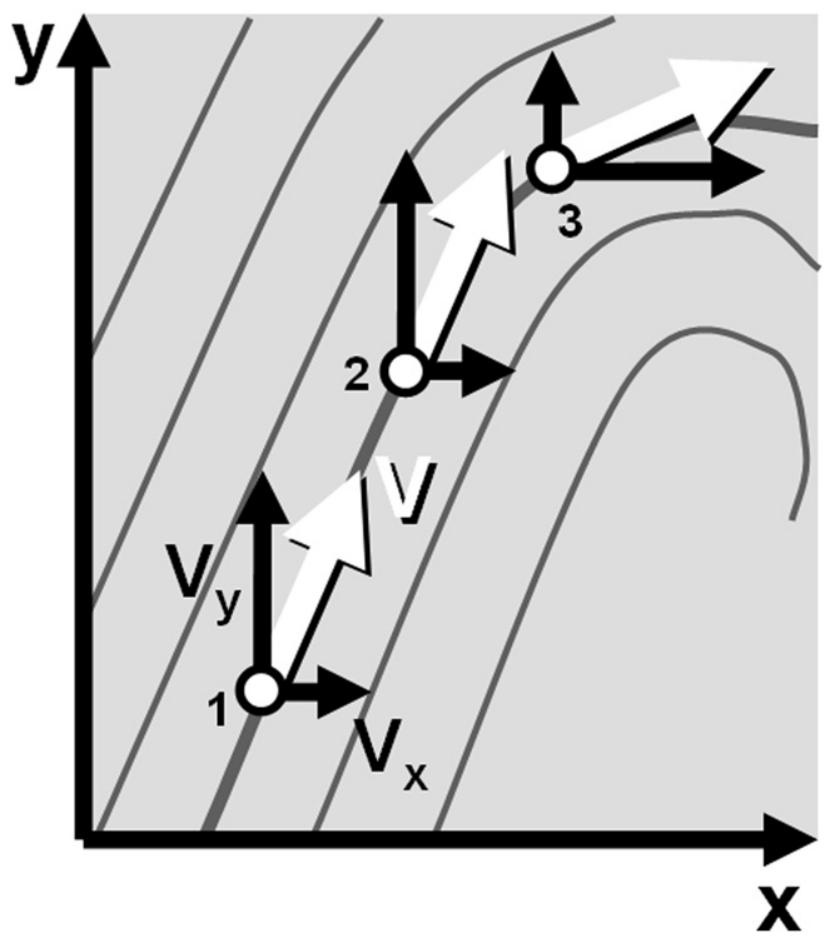

FIGURE 1. Definition of vorticity in 2 dimensions. Vorticity is a measure of the curl of streamlines (gray lines) of a fluid. According to equation 1, vorticity will be zero (laminar flow) if calculated between points 1 and 2, and it will be negative if calculated between points 2 and 3 (clockwise rotation). $v$, Flow velocity; $v x, v y, \mathrm{x}$ - and $\mathrm{y}$-components of the flow velocity.

them allowed us to obtain regional flow information (by means of PIV). Third, the software analyzed the left ventricular cavitary flow field and derived parameters to describe flow patterns. The maximal vortex area during diastole was given in percentages of the left ventricular area. If 2 vortices were present, the sizes were added. Estimates of energy dissipation (RS, VRS, and VPC) were retrieved. Flow patterns were visually assessed by interpreting a field of flow velocity vectors superimposed on the grey scale image loops. Color-coded images visualized the flow patterns averaged over the cardiac cycle.

\section{Statistical Analysis}

Continuous variables are presented as means \pm standard deviations. Differences between 2 groups were tested for significance by using the unpaired $t$ test. For more than 2 groups, analysis of variance with a Bonferroni post hoc test was used. Contingency tables were analyzed with a $\chi^{2}$ test. Mean standard deviations between readings were calculated to assess reproducibility of data analysis. Data were analyzed with SPSS 13.0 software (SPSS, Inc, Chicago, Ill).

\section{RESULTS}

Patient Data, Feasibility, and Reproducibility

Table 1 summarizes the characteristics of our study population. Patients were significantly older than the healthy subjects. No significant differences were found between the groups regarding sex distribution, body surface area, ejection fraction, or left ventricular end-diastolic volume. Left atrial area and mean mitral valve gradient were significantly larger in patients with prosthetic mitral valves.
TABLE 1. Clinical and echocardiographic characteristics of our study population

\begin{tabular}{lccc}
\hline & $\begin{array}{c}\text { Healthy } \\
\text { subjects } \\
(\mathbf{n}=\mathbf{9})\end{array}$ & $\begin{array}{c}\text { Patients with } \\
\text { prosthetic MVs } \\
(\mathbf{n}=\mathbf{1 0})\end{array}$ & $\begin{array}{c}\boldsymbol{P} \\
\text { value }\end{array}$ \\
\hline Age $(\mathrm{y})$ & $40 \pm 11$ & $72 \pm 6$ & .000 \\
BSA $\left(\mathrm{m}^{2}\right)$ & $1.82 \pm 0.29$ & $1.81 \pm 0.16$ & .972 \\
Male/female sex & $4 / 5$ & $5 / 5$ & .808 \\
EDWT septum $(\mathrm{mm})$ & $8.9 \pm 1.1$ & $11.7 \pm 2.2$ & .003 \\
EDWT lateral wall $(\mathrm{mm})$ & $9.3 \pm 1.3$ & $11.1 \pm 1.3$ & .009 \\
LV EDV/BSA $\left(\mathrm{mL} / \mathrm{m}^{2}\right)$ & $52.8 \pm 9.1$ & $50.9 \pm 8.7$ & .643 \\
LV EF $(\%)$ & $61.6 \pm 6.8$ & $59.7 \pm 7.6$ & .079 \\
LA area/BSA $\left(\mathrm{cm}^{2} / \mathrm{m}^{2}\right)$ & $9.1 \pm 1.3$ & $15.0 \pm 2.3$ & .000 \\
Mean MV gradient $(\mathrm{mm}$ Hg) & $0.8 \pm 0.3$ & $4.5 \pm 2.0$ & .000 \\
\hline$M V$, Mitral valve; $B S A$, body surface area; $E D W T$, end-diastolic wall thickness; $L V$, \\
left ventricular; $E D V$, end-diastolic volume; $E F$, ejection fraction; $L A$, left atrial.
\end{tabular}

Because of severe emphysema, acquisition of a 3-chamber view was not possible in 1 patient with a prosthetic mitral valve. Otherwise, offline analysis of left ventricular cavitary blood flow patterns was feasible in all subjects.

Parameters of vortex area and energy dissipation showed low intraobserver variability: standard deviations between 3 repeated readings were $3.7 \%, 6.0 \%, 5.7 \%$, and $6.2 \%$ for RS, VRS, VPC and vortex area, respectively.

\section{Flow Patterns in Healthy Subjects}

In all healthy subjects, we found a vortex that detaches from the anterior leaflet of the mitral valve early after the peak of the E-wave (Figure 2, A). This vortex rotates counterclockwise in the 4-chamber view and clockwise in the 3chamber view and fills almost the entire left ventricle by the end of the E-wave (Figure 2, B). With atrial contraction, late mitral inflow is directed more laterally and generates a second smaller vortex with equal rotation direction in the basal half of the left ventricle (Figure 2, C). With the beginning of systole, the rotating blood is redirected toward the outflow tract (Figure 2,D). Laminar flow is noted when blood leaves the left ventricle. Figure 2, $E$ and $G$, shows the spatial vorticity distribution of the left ventricle averaged over the cardiac cycle.

Quantitative parameters of energy dissipation are summarized in Table 2. RS and VPC values were significantly lower in healthy hearts than in patients with prosthetic valves, whereas the maximal vortex size did not differ between the groups.

\section{Flow Patterns of Bileaflet Valves in Anatomic Orientation}

General pattern. All patients had their bileaflet prostheses implanted in anatomic orientation (ie, with the hinge positions approximating commissures of the previous native valve). The 2 outer orifices generate jets directed toward the left ventricular septum and the lateral wall. In the 

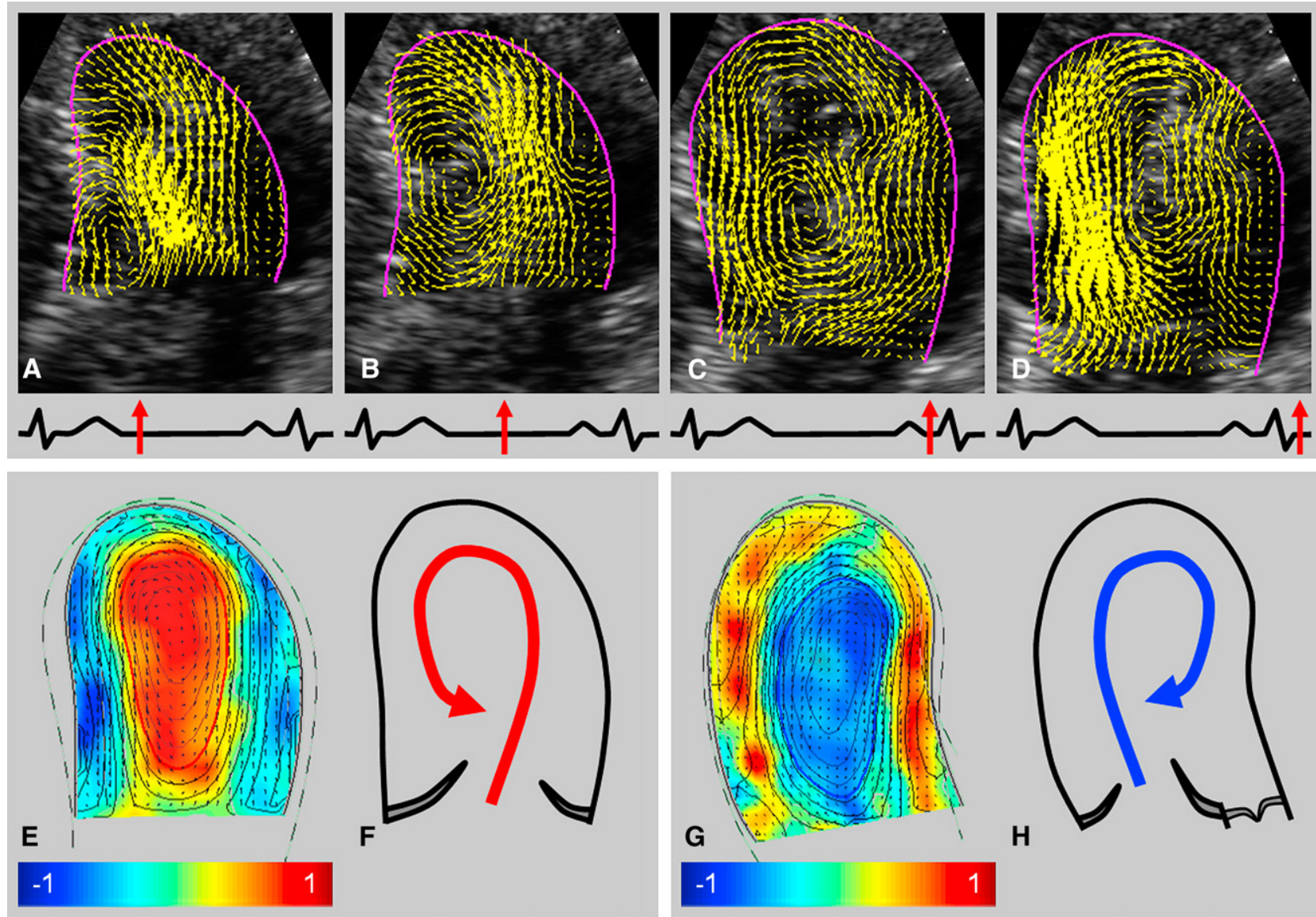

FIGURE 2. Flow pattern in a healthy left ventricle. Yellow arrows indicating the instantaneous local direction and velocity of blood flow are superimposed on a contrast-enhanced grayscale image of a 4-chamber view. A-D, See text for description of the temporal sequence. Use the electrocardiogram as time reference. Normalized regional vorticity in the plane of the 4-chamber view is averaged over the cardiac cycle. Counterclockwise rotation of the blood (positive vorticity) is color coded in red, and clockwise rotation is color coded (negative vorticity) in blue. Schematic drawings of the dominating flow path as observed during diastole are shown. $\mathrm{G}$ and $\mathrm{H}$, Same display as $\mathrm{E}$ and $\mathrm{F}$ but a 3-chamber view. Note that in images $\mathrm{E}$ and $\mathrm{F}$, the central vortex is surrounded by a color rim indicating an opposite vorticity. This is explained by blood velocities, which gradually increase from the endocardium toward the vortex structure. Such a velocity gradient can be interpreted as vorticity and is thus color coded.

4-chamber view the contrast bubbles of the septal jet reach the apex first, whereas those of the lateral jet travel slower (Figure 3, A). Subsequently, during the deceleration of the E-wave, the septal jet becomes dominant (Figure 3, B) and forms a major vortex during the A-wave, rotating clockwise in the 4-chamber view (Figure 3,C). Thus with the beginning of systole, outflowing blood crosses the previous inflow area (Figure 3,D).
In general, the resulting vorticity pattern in hearts of patients with bileaflet valves is opposite to that seen in healthy hearts: a large clockwise rotating vortex can be seen in blue in the 4-chamber view (Figure 3,E) whereas the 3-chamber view shows a counterclockwise rotating vortex in red (Figure 3, $G$ ).

Influence of left ventricular geometry. Two patients with bileaflet mitral valve prostheses had a marked midseptal

TABLE 2. Characteristics of the left ventricular vortical flow in healthy hearts and hearts of patients with prosthetic mitral valves

\begin{tabular}{|c|c|c|c|c|c|c|c|c|c|}
\hline & Healthy hearts & $\begin{array}{l}\text { All prosthetic } \\
\text { valves }\end{array}$ & $P$ value* & Bileaflet valves & $\boldsymbol{P}$ value $\dagger$ & $\begin{array}{c}\text { Bioprosthetic } \\
\text { valves }\end{array}$ & $\boldsymbol{P}$ value $\dagger$ & Tilting-disc valve & $\boldsymbol{P}$ value $\dagger$ \\
\hline VA $(\%)$ & $50.3 \pm 5.9$ & $53.5 \pm 13.6$ & .352 & $50.6 \pm 16.7$ & 1.000 & $56.9 \pm 8.5$ & 1.000 & $56.2 \pm 15.2$ & 1.000 \\
\hline RS & $1.62 \pm 0.43$ & $2.36 \pm 0.71$ & .000 & $2.33 \pm 0.92$ & .037 & $2.43 \pm 0.48$ & .034 & $2.28 \pm 0.16$ & .941 \\
\hline VRS & $0.43 \pm 0.10$ & $0.52 \pm 0.16$ & .052 & $0.50 \pm 0.16$ & 1.000 & $0.53 \pm 0.18$ & .828 & $0.64 \pm 0.09$ & .315 \\
\hline VPC & $0.72 \pm 0.24$ & $1.20 \pm 0.51$ & .000 & $1.20 \pm 0.60$ & .041 & $1.22 \pm 0.49$ & .076 & $1.22 \pm 0.29$ & .743 \\
\hline
\end{tabular}

Relative pulsatile vorticity strength $(R S)$, vortex relative pulsatile vorticity strength $(V R S)$, and vortex pulsation correlation $(V P C)$ are parameters of flow pattern-dependent energy dissipation in the left ventricle. See the text for further details. $V A$, Vortex area as a percentage of the left ventricular area. $P$ values versus healthy hearts: $* t$ test $\dagger$ analysis of variance post hoc test (Bonferroni correction). 

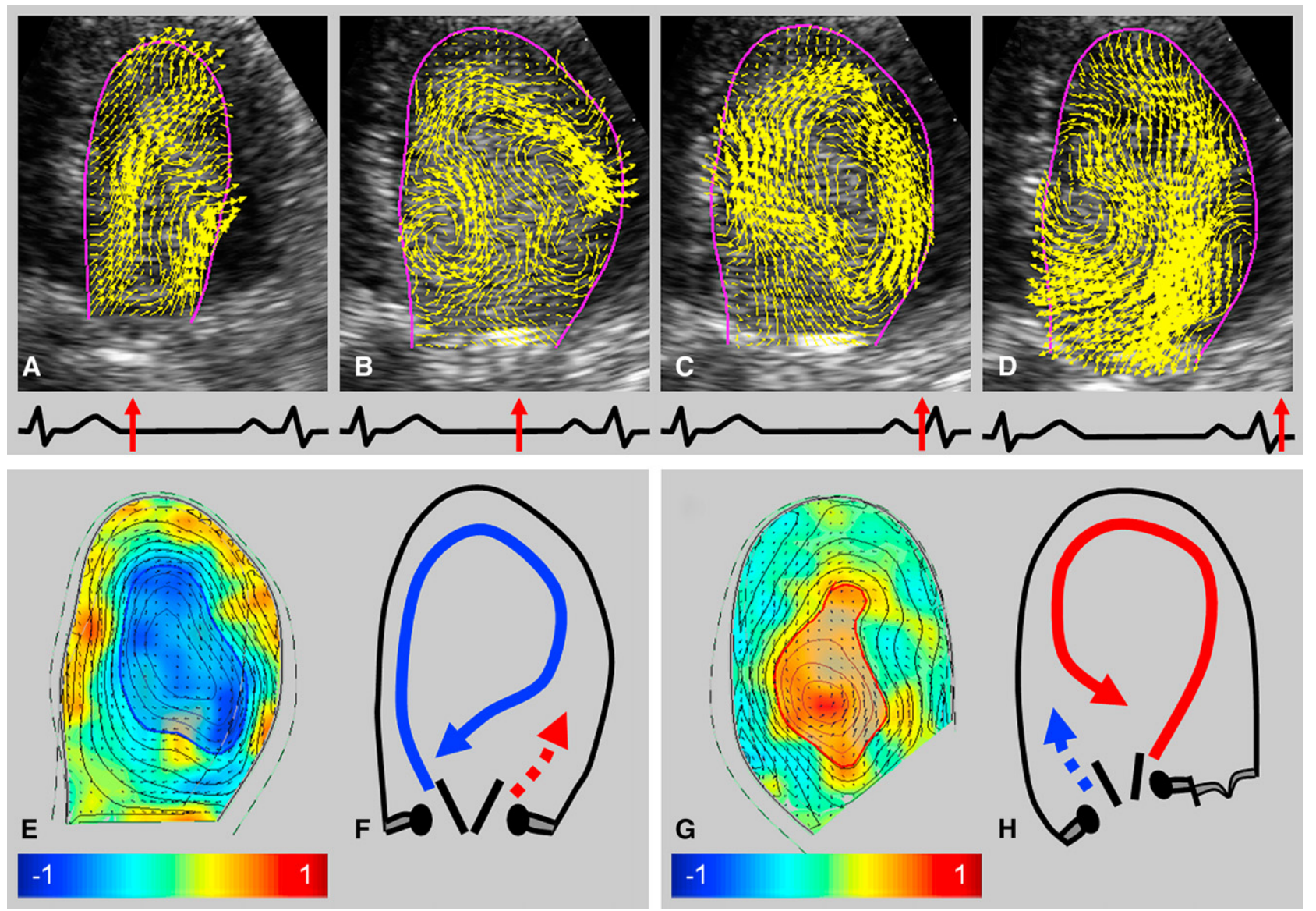

FIGURE 3. General flow pattern in patients with bileaflet mitral valve prosthesis in anatomic orientation. There is a similar display as in Figure 2. See the text for a detailed description.

hypertrophy (maximal wall thickness, 14 and $15 \mathrm{~mm}$, respectively). In those patients the septal jet did not reach the apex directly but was redirected toward the lateral wall. From there, it split into 2 streams forming 2 counterrotating vortices: a clockwise rotating vortex in the basal half of the ventricle and a second, counterclockwise rotating vortex in the apex (Figure 4, $A$ and $C$, upper panels).

With the beginning of systole, outflowing blood again crosses the previous inflow area (Figure 4, $B$, upper panel). Figure 4, $D$ (upper panel), provides a schematic drawing of the main flow paths.

Parameters of energy dissipation (RS and VPC) were significantly higher in patients with bileaflet valves than in healthy subjects (Table 2). Compared with patients with bileaflet valves with the general flow pattern, RS tended to be particularly high in those with altered left ventricular geometry $(1.98 \pm 0.82$ vs $2.85 \pm 0.90, P=.171)$.

\section{Flow Patterns of Bioprosthetic Valves}

General pattern. The flow through the bioprosthesis generates a central jet directed toward the apex (Figure 5, A). It generates a vortex ring that is visualized as a vortex pair in a 2-dimensional image (Figure 5, B). With atrial contraction, the same flow pattern is reinforced. At the beginning of systole (Figure 5,C), the symmetry of the flow pattern is lost, the posterolateral vortex part becomes dominant, and the outflowing blood crosses the previous inflow area again (Figure 5,D). This dominance of the posterolateral vortex part might also be noted in the time-averaged color-coded representation of left ventricular vorticity from both apical views (Figure 5, $E$ and $G$ ). Influence of valve position and left ventricular geometry. One patient had a marked anteroseptal position of the bioprosthesis, resulting in an inflow jet toward the anterior septum. Similar to the abovementioned bileaflet valves, this jet is then redirected toward the lateral wall by the hypertrophied midseptum (Figure 4, $A$, lower panel) and forms 2 vortices (Figure 4, $B$, lower panel). Figure 4, $C$ (lower panel), shows the time-averaged vorticity in the 4-chamber view, and Figure 4, $D$ (lower panel), provides a schematic drawing of the main flow paths.

In hearts of patients with bioprosthetic valves, parameters of energy dissipation were higher than in hearts of healthy subjects. RS showed a significant difference, and VPC showed a trend (Table 2). Energy dissipation was particularly high in the patient with altered left ventricular geometry (RS, $2.90 \pm 0.48$; VPC, $1.76 \pm 0.66$ ). 

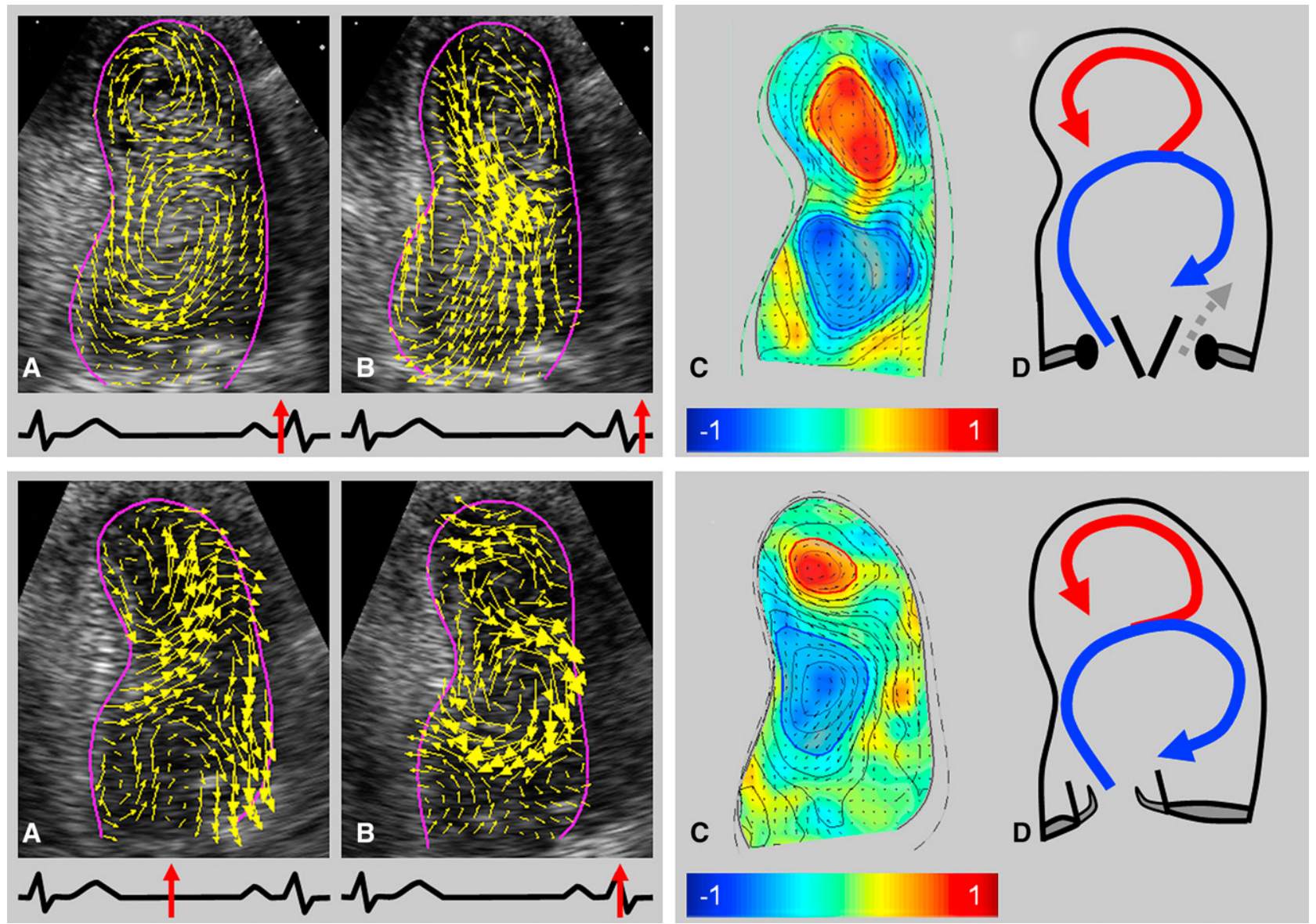

FIGURE 4. Flow patterns in patients with bileaflet valves in anatomic orientation (upper panels) and bioprosthetic valves (lower panels) and marked midseptal hypertrophy. A and B, See text for detailed description. Use the electrocardiogram as time reference. Normalized regional vorticity in the plane of the 4-chamber view is averaged over 1 cardiac cycle. Schematic drawings of the dominating flow path as observed during diastole in the 4-chamber view are shown.

\section{Flow Pattern of a Tilting-Disc Valve With Anterior Orientation of the Greater Orifice}

In our patient with a single-disc prosthesis, the majority of the inflow is directed through the greater orifice toward the septum and then redirected by the hypertrophied ventricular septum to form a large, clockwise rotating vortex filling the basal two thirds of the ventricle and a small, counterclockwise rotating vortex in the anterior apex (Figure 6, A). Similar to the other prosthetic valves, the outflowing blood crosses the previous inflow area (Figure $6, B)$. Likewise, parameters of energy dissipation are higher compared with those seen in healthy hearts (Table 2).

\section{DISCUSSION}

\section{Flow Patterns in Healthy Hearts}

In our study we found vortical flow patterns in the left ventricle, detaching from the mitral valve during mitral inflow (Figure 2). Based on the 2-dimensional data obtained in the 3 apical image planes, we could conclude that a 3-dimensional, torus-like vortex structure (similar to a donut or a smoke ring puffed out by a cigarette smoker) enters the left ventricle during the beginning of early diastole. Because of the asymmetry of the left ventricular inflow tract, the anteroseptal side of the vortex formation becomes dominant and persists in the center of the cavity, leading to the described rotational motion of the blood in the ventricle, whereas the smaller posterior part of the vortex diminishes rapidly. This is in concordance with prior physical and numeric modeling ${ }^{19-22}$ and supported by in vivo studies with color Doppler imaging ${ }^{23}$ and MRI. ${ }^{2-4}$

During atrial contraction, we found a more laterally directed mitral inflow forming a second vortex structure at the basal half of the ventricle. This is in concordance with MRI observations reported by Fyrenius and colleagues, ${ }^{4}$ who similarly describe 2 different pathways of the inflowing blood during early and late filling.

At the very end of diastole, a single large vortex is present, and blood is smoothly redirected toward the outflow tract for 

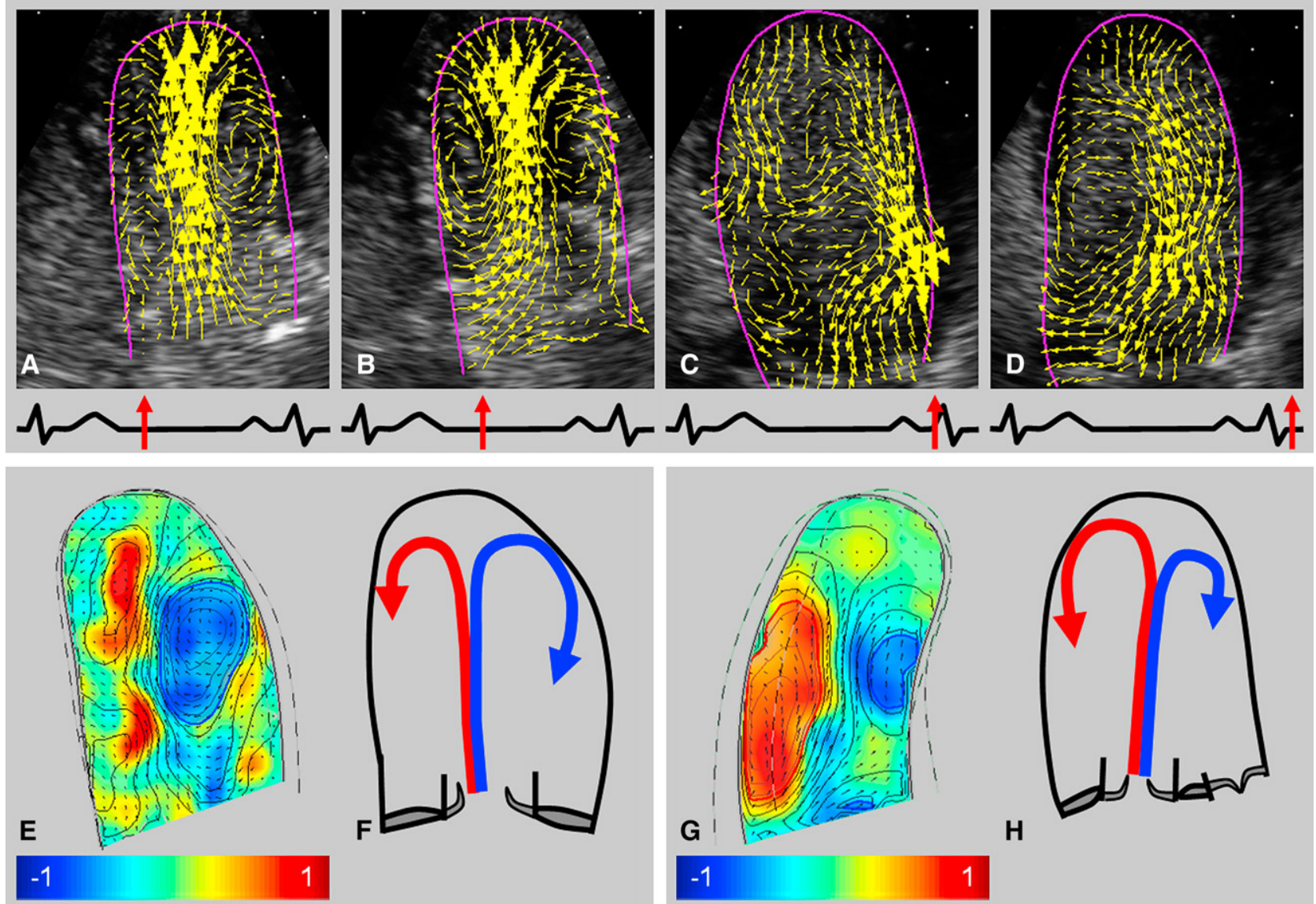

H
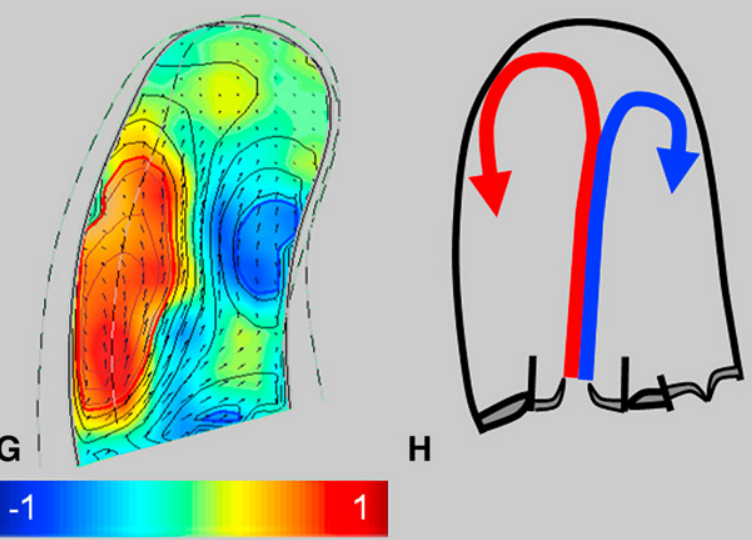

FIGURE 5. General flow pattern in patients with bioprosthetic valves. There is a similar display as in Figure 2. See the text for a detailed description.

the following systole, as predicted based on numeric modeling by others. ${ }^{20,21}$

\section{Bileaflet Valve in Anatomic Orientation}

The nonphysiologic inflow through a bileaflet valve leads to a completely different and more complex flow pattern in the left ventricle (Figure 3). In most of our patients, the inflow through the septal orifice was dominant and resulted in a major vortex rotating in the opposite direction compared with that seen in healthy hearts. This is partially supported by prior in vitro simulations ${ }^{24-26}$ and animal experiments $^{27-29}$ and might be explained by asymmetry of the
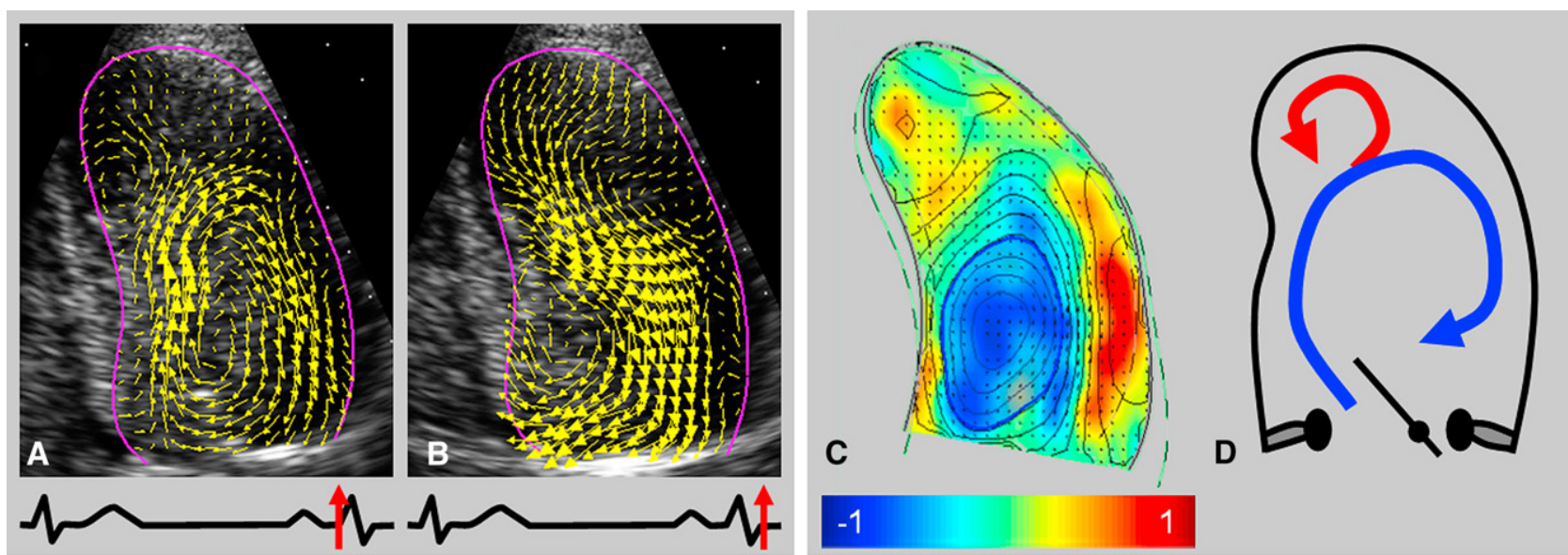

FIGURE 6. Flow pattern in a patient with a tilting-disc valve with anterior orientation of the greater orifice. This is the same principle of display as in Figure 4 . See the text for a detailed description. 
left ventricular inflow tract. Because the septal inflow chamber, which includes the left ventricular outflow tract, is larger than the posterior one, the mitral inflow penetrates the ventricle mainly through the septal orifice. ${ }^{28}$ Asynchronous opening of the leaflets ${ }^{26}$ or asymmetry of the atrial flow $^{5,6,25}$ might further contribute.

Our measurements indicate for the first time, that intracavitary blood flow in patients with bileaflet valve prostheses is less energy efficient than in healthy hearts. Higher energy dissipation can be explained by the nonphysiologic formation of a major vortex rotating in the opposite direction compared with that seen in healthy hearts and the outflowing blood crossing the inflow tract during systole.

Our results show that unfavorable left ventricular geometry further disturbs vortex formation. This has not been described previously because experimental studies assume "normal" ventricles. Nevertheless, with the observation of 2 counterrotating vortices in the apical and basal halves of the ventricle, we can show for the first time a human in vivo correlate to the color Doppler patterns described by Van Rijk-Zwikker and coworkers ${ }^{28}$ in pigs.

\section{Bioprosthetic Valve}

In concordance with the numeric simulations of Pedrizzetti and Domenichini, ${ }^{10}$ we observed in patients with bioprosthetic valves a completely symmetric inflow pattern leading to a symmetric vortex ring (Figure 5). At the end of diastole, this symmetry is lost, and the part of the vortex ring that becomes dominant rotates in the opposite direction compared with that seen in healthy hearts.

Our data indicate that the flow pattern in bioprosthetic valves also results in higher energy dissipation. This might be explained by the division of the blood stream in counterrotating parts that leads to a higher loss of kinetic energy than in the single vortex of a normal heart. Position of the valve and, similar to bileaflet prosthesis, left ventricular geometry markedly influence the flow pattern. Our data help us understand the previous color Doppler observations of Maire and associates, ${ }^{30}$ who described unusual flow patterns in patients with an eccentric bioprosthetic valve position.

\section{Tilting-Disc Valve With Anterior Orientation of the Greater Orifice}

Our findings in the patient with a tilting-disc prosthesis are in concordance with previous Doppler- or MRI-based publications. ${ }^{30-32}$ The presence of a hypertrophied left ventricular septum explains the formation of the additional counterrotating vortex at the apex (Figure 6). Single tilting-disc prostheses are rarely used in the mitral position nowadays. Based on our experiences, however, we hypothesize that the normal direction of inflow vortex formation and the separation of inflow and outflow tracts might be best simulated by a mitral tilting-disc prosthesis with the larger orifice in a posterior orientation. ${ }^{30-32}$ To what extent this might counterbalance the disadvantages of this type of valve replacement therapy remains to be determined.

\section{Clinical Considerations}

It has been assumed based on numeric simulations and in vitro and in vivo experiments, as well as color Doppler and MRI measurements, that prosthetic heart valves seriously disturb intraventricular flow patterns. ${ }^{10,19,24-32}$ The application of PIV to echocardiography allows, for the first time, direct visualization and, in part, quantification of those patterns and enables estimation of energy dissipation in the left ventricle by means of a noninvasive technique.

Numeric simulations of energy dissipation support the thesis that the natural left ventricle is optimized to forward the blood with the lowest energy loss possible. ${ }^{11}$ A discontinuous blood pump can work most efficiently if the momentum of the blood is maintained. A linear arrangement of heart chambers would require constant deceleration and acceleration of the blood and would waste kinetic energy. In contrast to any linear arrangement of heart chambers, it is more energy efficient to have adjacent inflow and outflow tracts and a diastolic vortex formation, which stores energy and redirects the blood stream while maintaining its kinetic energy. ${ }^{11}$

To what extent vortex formation contributes to an optimization of cardiac performance is subject to debate. ${ }^{33}$ Some authors assume that accelerating blood accounts only for approximately $2 \%$ of the energy consumption of a healthy heart. ${ }^{34}$ Pedrizzetti and Domenichini ${ }^{10}$ showed in a numeric simulation that even slight changes in mitral valve position can result in energy dissipation in the range of $15 \%$. A counterrotating vortex or more complex flow-pattern changes are likely to result in even higher energy loss. It might therefore be relevant to optimize intracavitary blood flow after valve replacement, particularly in dysfunctional ventricles. We believe that this new technique of echocardiographic PIV can contribute to a better understanding of hemodynamic consequences of heart valve surgery and thus to an optimization of such a therapy.

\section{Limitations of the Study}

The current echocardiographic PIV method is 2 dimensional and thus has limits regarding the analysis of the 3-dimensional structure of flow patterns. This inherent problem of clinical echocardiographic analysis can currently not be solved because of the low temporal and spatial resolution of echocardiographic 3-dimensional imaging technology. We are confident, however, that merging information from 3 apical views is sufficient to recognize larger 3-dimensional structures in the intracavitary flow of the left ventricle. This is supported by the fact that the results of our data interpretation are in accordance with mathematic modeling and true 3-dimensional MRI measurements of cavitary flow. Future 
developments in 3-dimensional echocardiography might further improve echocardiographic PIV.

Thus far, flow-pattern analysis is descriptive and at best semiquantitative. The relation between flow patterns and energy dissipation is extremely complex and difficult to express in a few simple, clinically useful numbers. Further studies are needed to better understand these relationships and to offer quantitative measures for intracavitary blood flow analysis.

Ventricular shape changes caused by age, previous mitral valve disease, or removal of the subvalvular apparatus were not considered. The study was not powered to investigate possible relations between valve size and flow patterns. Our data suggest, however, that the presence of a mitral valve prosthesis dominates intracavitary flow-pattern developments and is only modulated by left ventricular geometry. Outcome data are needed to evaluate the clinical relevance of our findings.

\section{Conclusion and Outlook}

For the first time, we present a description of normal intraventricular blood flow patterns and their changes caused by mitral valve surgery based on noninvasive echocardiographic PIV. The technique was feasible in the clinical setting and allowed clear differentiation of normal from disturbed flow patterns after mitral valve replacement, as well as quantification of left ventricular energy dissipation.

Future applications of echocardiographic PIV might comprise the study of postoperative left ventricular flow characteristics to optimize bioprosthetic and mechanical heart valve design. Preoperative PIV could help to select most optimal implantation techniques, such as valve replacement with or without preservation of the subvalvular apparatus, or to optimize implantation for a specific pathology, such as postinfarction patients or hearts with septal hypertrophy. Flow investigations by PIV could also improve valve reconstruction strategies or help to optimize other surgical cardiovascular interventions, such as Fontan operations. Pathophysiologic knowledge could be gained on the relation between left ventricular and left atrial flow characteristics and the rate of thromboembolic events.

We therefore conclude that echocardiographic PIV has potential clinical importance for the analysis of cardiac function and guidance of cardiosurgical interventions.

\section{References}

1. Gharib M, Kremers D, Koochesfahani MM, Kemp M. Leonardo's vision of flow visualization. Exp Fluids. 2002;33:219-23.

2. Kim WY, Walker PG, Pedersen EM, Poulsen JK, Oyre S, Houlind K, et al. Left ventricular blood flow patterns in normal subjects: a quantitative analysis by three-dimensional magnetic resonance velocity mapping. J Am Coll Cardiol. 1995;26:224-38.

3. Wigström L, Ebbers T, Fyrenius A, Karlsson M, Engvall J, Wranne B, et al. Particle trace visualization of intracardiac flow using time-resolved 3D phase contrast MRI. Magn Reson Med. 1999;41:793-9.
4. Fyrenius A, Wigström L, Bolger AF, Ebbers T, Ohman KP, Karlsson M, et al. Pitfalls in Doppler evaluation of diastolic function: insights from 3-dimensional magnetic resonance imaging. J Am Soc Echocardiogr. 1999; 12:817-26.

5. Kilner PJ, Yang GZ, Wilkes AJ, Mohiaddin RH, Firmin DN, Yacoub MH. Asymmetric redirection of flow through the heart. Nature. 2000;404:759-61.

6. Fyrenius A, Wigström L, Ebbers T, Karlsson M, Engvall J, Bolger AF. Three dimensional flow in the human left atrium. Heart. 2001;86:448-55.

7. Yang GZ, Mohiaddin RH, Kilner PJ, Firmin DN. Vortical flow feature recognition: a topological study of in vivo flow patterns using MR velocity mapping. $J$ Comput Assist Tomogr. 1998;22:577-86.

8. Kvitting JP, Ebbers T, Wigström L, Engvall J, Olin CL, Bolger AF. Flow patterns in the aortic root and the aorta studied with time-resolved, 3-dimensional, phase contrast magnetic resonance imaging: implications for aortic valve-sparing surgery. J Thorac Cardiovasc Surg. 2004;127:1602-7.

9. Batchelor GK. The theory of homogeneous turbulence. Cambridge (UK): Cambridge University Press; 1953.

10. Pedrizzetti G, Domenichini F. Nature optimizes the swirling flow in the human left ventricle. Phys Rev Lett. 2005;95:108101.

11. Krueger $P$, Gharib M. The significance of vortex ring formation to the impulse and thrust of a starting jet. Phys Fluids. 2003;15:1271-81.

12. Milo S, Rambod E, Gutfinger C, Gharib M. Mitral mechanical heart valves: in vitro studies of their closure, vortex and microbubble formation with possible medical implications. Eur J Cardiothorac Surg. 2003;24:364-70.

13. Rahimtoola SH. Choice of prosthetic heart valve for adult patients. J Am Coll Cardiol. 2003;41:893-904.

14. Cen YY, Glower DD, Landolfo K, Lowe JE, Davis RD, Wolfe WG, et al Comparison of survival after mitral valve replacement with biologic and mechanical valves in 1139 patients. J Thorac Cardiovasc Surg. 2001;122: 569-77.

15. Adrian RJ. Particle-imaging technique for experimental fluid mechanics. Annu Rev Fluid Mech. 1991;23:261-304.

16. Zheng H, Mukdadi O, Hertzberg J, Shandas R. Advantages in using multi-frequency driving ultrasound for optimizing echo particle image velocimetry techniques. Biomed Sci Instrum. 2004;40:371-6.

17. Sengupta PP, Khandheria BK, Korinek J, Jahangir A, Yoshifuku S, Milosevic I, et al. M. Left ventricular isovolumic flow sequence during sinus and paced rhythms: new insights from use of high-resolution Doppler and ultrasonic digital particle imaging velocimetry. J Am Coll Cardiol. 2007;49:899-908.

18. Hong GR, Pedrizzetti G, Tonti G, Li P, Kim JK, Wei Z, et al. Characterization and quantification of vortex flow in the human left ventricle by contrast echocardiography using vector particle image velocimetry. JACC Cardiovasc Imaging. 2008 1:705-17.

19. Bellhouse BJ. Fluid mechanics of a model mitral valve and left ventricle. Cardi ovasc Res. 1972;6:199-210.

20. Bolzon G, Zovatto L, Pedrizzetti G. Birth of three-dimensionality in a pulsed jet through a circular orifice. J Fluid Mech. 2003;493:209-18.

21. Domenichini F, Pedrizzetti G, Baccani B. Three-dimensional filling flow into a model left ventricle. J Fluid Mech. 2005;539:179-98.

22. Domenichini F, Querzoli G, Cenedese A, Pedrizzetti G. Combined experimental and numerical analysis of the flow structure into the left ventricle. $J$ Biomech. 2007;40:1988-94.

23. Rodevand O, Bjornerheim R, Edvardsen T, Smiseth OA, Ihlen H. Diastolic flow pattern in the normal left ventricle. J Am Soc Echocardiogr. 1999;12: 500-7.

24. Schoephoerster RT, Chandran KB. Velocity and turbulence measurements past mitral valve prostheses in a model left ventricle. J Biomech. 1991;24: 549-62.

25. Garitey V, Gandelheid T, Fuseri J, Pélissier R, Rieu R. Ventricular flow dynamic past bileaflet prosthetic heart valves. Int J Artif Organs. 1995;18: 380-91.

26. Pierrakos O, Vlachos PP, Telionis DP. Time-resolved DPIV analysis of vortex dynamics in a left ventricular model through bileaflet mechanical and porcine heart valve prostheses. J Biomech Eng. 2004;126:714-26.

27. Jones M, Eidbo EE. Doppler color flow evaluation of prosthetic mitral valves: experimental epicardial studies. J Am Coll Cardiol. 1989;13:234-40.

28. Van Rijk-Zwikker GL, Delemarre BJ, Huysmans HA. The orientation of the bileaflet CarboMedics valve in the mitral position determines left ventricular spatial flow patterns. Eur J Cardiothorac Surg. 1996;10:513-20.

29. Mächler H, Perthel M, Reiter G, Reiter U, Zink M, Bergmann P, et al. Influence of bileaflet prosthetic mitral valve orientation on left ventricular flow-an 
experimental in vivo magnetic resonance imaging study. Eur J Cardiothorac Surg. 2004;26:747-53.

30. Maire R, Ikram S, Odemuyiwa O, Groves PH, Lo SV, Banning AP, et al. Abnormalities of left ventricular flow following mitral valve replacement: a color flow Doppler study. Eur Heart J. 1994;15:293-302.

31. Pop G, Sutherland GR, Roelandt J, Vletter W, Bos E. What is the ideal orientation of a mitral disc prosthesis? An in vivo haemodynamic study based on color flow imaging and continuous wave Doppler. Eur Heart J. 1989;10:346-53.
32. Mächler H, Reiter G, Perthel M, Reiter U, Bergmann P, Zink M, et al. Influence of a tilting prosthetic mitral valve orientation on the left ventricular flow-an experimental in vivo magnetic resonance imaging study. Eur J Cardiothorac Surg. 2007;32:102-7.

33. Watanabe H, Sugiura S, Hisada T. The looped heart does not save energy by maintaining the momentum of blood flowing in the ventricle. Am J Physiol Heart Circ Physiol. 2008;294:H2191-6.

34. Schmidt RF, Lang F, Thews G. Physiologie des Menschen mit Pathophysiologie. Heidelberg: Springer; 2004. 